\title{
Cerebral evoked potentials in patients with dissociated sensory loss
}

\author{
A. M. HALLIDAY ${ }^{1}$ AND G. S. WAKEFIELD ${ }^{2}$
}

From the National Hospital, Queen Square, London

After stimulation of a peripheral nerve in man a small evoked response can be recorded from a surface electrode placed over the contralateral postcentral gyrus, as was first shown by Dawson (1947a). In normal healthy subjects the response is only a few microvolts in size, and its detection is uncertain unless use is made of a summation technique of the kind which Dawson himself subsequently introduced (Dawson, 1954). Recorded in this way, however, a clear response can be obtained from any normal healthy subject. It has a latency, constant to within a few milliseconds, characteristic for the site of stimulation employed. Furthermore, while quite marked individual variations may occur in the amplitude and wave-form recorded from different subjects, the early components of the response appear to change minimally in the same subject from time to time, provided that the conditions of the experiment are not materially altered.

Not many investigations of the effect of neurological disease on this cortical response have so far been published. Dawson (1947b) showed that in a case of myoclonic epilepsy the amplitude of the potential was five to 10 times larger than normal but that the latency and duration of the response was the same as that in healthy subjects. Alajouanine, Scherrer, Barbizet, Calvet, and Verley (1958) were able to record the responses in 26 out of a series of 50 patients suffering from disturbances of somatic sensation due to a large variety of causes. These included 10 cases with cortical lesions (two with a thalamic syndrome), five cases with lesions of brainstem or cord, three with root lesions, one case of congenital insensitivity to pain, and seven cases of hysterical hemianaesthesia. The limitations of their recording technique did not allow them to study the latency, wave-form, or distribution of the response and they were concerned only with amplitude changes. They reported no cases in which the potential was larger than normal and found it to be of normal amplitude in the patients with hysterical anaesthesia or congenital indifference to pain. In

\footnotetext{
${ }^{1}$ Member of the external staff of the Medical Research Counci
} 'In receipt of a grant from the Institute of Neurology the other cases it was apparently unaffected if the sensory impairment was mild, but was either absent or appeared only with stronger stimulation than normal if the sensory loss was severe; this was so whether the lesion was central or peripheral. They stated that the sensory loss had to be very pronounced before the cortical response disappeared. In such cases, the stimulus could still be felt but had a different quality when compared with the stimulus on the healthy side. They suggested either that the afferent volley might still be arriving at other cortical areas or that the volley might no longer be sufficiently synchronous to give a clear response.

They commented particularly on two cases with lesions affecting the spinothalamic tract on one side. In these patients, the response on the affected side was definitely smaller for near-threshold stimuli, but with stronger stimuli appeared similar to the response from the healthy side. The diminution in amplitude they took to indicate that some of the impulses responsible for the cortical response follow the spinothalamic route.

Giblin (1960), using Dawson's summation technique, examined a series of patients with different types of sensory loss, including five cases with lesions of the peripheral nerves and posterior roots, seven cases with lesions of the cord, and 14 cases with a lesion involving the parietal lobe of one hemisphere. In the last group the results correlated with the severity of the sensory impairment (as in the cases of Alajouanine et al.). Where sensory loss was slight (three cases) the potentials appeared normal or slightly delayed; but where it was severe ( 10 cases) the evoked potential from the damaged hemisphere was absent or markedly abnormal. In the five patients with peripheral nerve or posterior root lesions, the evoked response to stimulation of the affected limb was greatly reduced or absent. The results obtained with the seven patients with cord lesions suggested that the cerebal evoked potential is dependent on intact posterior column function (as judged clinically) and is not abolished by lesions affecting the ascending pathways in the antero-lateral columns. 
In the present investigation we have studied cerebral evoked potentials in 14 patients with various types of dissociated sensory loss due to lesions of the afferent tracts. In two the lesion was at brain-stem level; in the remainder the lesion was of the spinal cord. A preliminary report of these cases, here to be described in detail, has already appeared (Halliday and Wakefield, 1962).

\section{MATERIAL}

Fourteen patients were investigated between August 1961 and March 1962. They were a consecutive series of cases, unselected except in so far as all had loss of appreciation of one or more sensory modalities with clinical evidence of a lesion involving the long ascending tracts. Table I

\section{TABLE I}

CLASSIFICATION OF PATIENTS ACCORDING TO SENSORY LOSS

Group Age of Sex Diagnosis

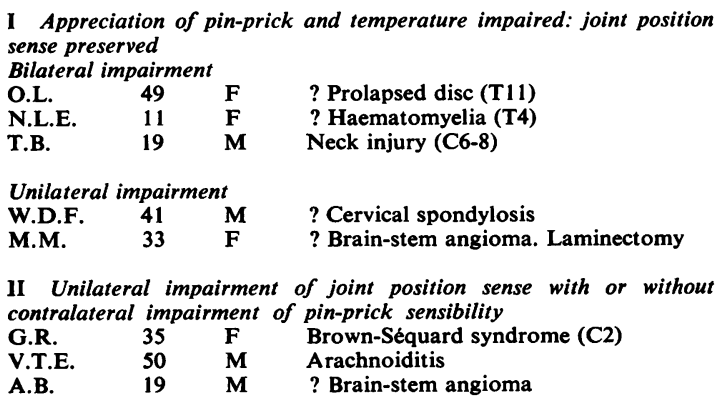

III Bilateral impairment of joint position sense: appreciation of pinprick preserved

$\begin{array}{llll}\text { C.K. } & 18 & \text { F } & \text { Disseminated sclerosis }\end{array}$

A.V.G. $\quad 59 \quad$ M $\quad$ Disseminated sclerosis

L.S.D. $\quad 56 \quad M \quad$ Meningioma (T4)

IV Partial recovery after bilateral impairment of joint position sense with or without residual bilateral impairment of pain and temperature sense

M.H. 49 F $6 / 12$ after removal of meningioma (T3)

F.P. $\quad 64 \quad F \quad 3 / 52$ after removal of meningioma (T4)

J.M. $\quad 55 \quad M \quad$ Cervical spondylosis

gives details of the cases, divided into four groups according to the type of sensory loss found on clinical examination, together with the proved or probable pathological diagnosis for each patient. It will be seen that a wide variety of ages are represented in all except the last group, that each group contains both male and female patients, and that though (as might be expected) certain diagnoses tend to be associated with a particular type of sensory loss, each type of sensory loss includes patients with more than one diagnosis. Any finding common to the members of one group is therefore unlikely to depend on factors other than the common sensory deficit. It will be noted that we have deliberately avoided using touch sensibility or two-point discrimination as a criterion for judging sensory loss of the fine discriminative type and have relied solely on joint-position sense. This has been done on the grounds that the latter is an uncomplicated index of the integrity of posterior column function, while touch sensation may be affected in lesions of either pathway (Rose and Mountcastle, 1959).

\section{METHODS}

Stimuli were applied by a two-channel thyratron stimulator which delivered condenser discharges (time constant either 0.5 or $1.25 \mathrm{msec}$.) through an isolating transformer. Stimuli to the upper limb were delivered to the index and middle fingers through ring electrodes, two on each finger, the more proximal electrode being the cathode. Stimuli were applied over the anterior tibial nerve at the ankle through a silver disc electrode, with a larger E.C.G.-type electrode strapped behind the medial malleolus as anode. In one patient (case 11) stimuli were delivered through ring electrodes to the second and third toes of each foot. An earth electrode was strapped to one limb.

All stimuli were delivered at a rate of 1 per second. At the beginning of the session the strength of stimulus just felt by the patient was recorded as the electrical threshold. These values are shown for each patient in Table II. The stimulus used to evoke a cortical potential was never less than twice this threshold intensity, except in two patients In one (case 7), the threshold was already so high that we were unable to deliver twice this stimulus strength witho the stimulator available. In the other (case 6), who had ag raised threshold on the side corresponding to loss of posterior column-type sensation, the patient was unableo to tolerate a stimulus of more than 1.4 times threshold on this side, as it became excessively unpleasant. In threes cases it was necessary to use the longer time constant stimulus to obtain the subjective threshold (Table II).

The evoked potential was recorded by means of Dawson's electromechanical averaging device (Dawson, 1954). Chloridized silver button electrodes (diameter $0.75 \mathrm{~cm}$.) were attached to the scalp with collodion. An active electrode was placed over the surface marking of the hand or foot area of the postcentral gyrus on each side, and the recording was made with respect to a similar, indifferent electrode placed $7 \mathrm{~cm}$. more anteriorly on the same side. An upward deflection on the display indicates negativity of the active electrode with respect to the indifferent electrode. Potentials from the two sides of the head were fed through two conventional E.E.G. amplifiers (time constant: $0 \cdot 2 \mathrm{sec}$. H.F. response: $3 \mathrm{~dB}$ down at $500 \mathrm{c} / \mathrm{s}$ ) to the averager.

The cerebral evoked response, measured through the scalp in healthy subjects, has an amplitude of the order of 3 to $5 \mu \mathrm{V}$, which is very near to the noise level of the recording amplifier (about 1 to $2 \mu \mathrm{V}$ ). It is in any case swamped by the far larger potentials of the electroencephalogram, or by any electromyographic activity in the scalp muscles. The averaging technique improves the resolution of the recording system by discriminating the response, which occurs regularly after each stimulus with a constant latency, from the randomly occurring background activity, which is not systematically related to the 
stimulus. Dawson's averager achieves this by using the instantaneous voltages for 62 ordinates spaced over $100 \mathrm{msec}$. to charge 62 capacitors connected to the studs on a circular rotating switch. The stimulus is always given when the wiper of the switch has reached a particular stud, so that any systematically occurring response produces a charge which summates steadily with successive stimuli in a particular capacitor or group of capacitors.

The averager has two channels which were used either to record the activity on the two sides of the head for the $10 \mathrm{msec}$. preceding and the $90 \mathrm{msec}$. succeeding the unilateral stimulus, or gated sequentially with a $10 \mathrm{msec}$. overlap to record the activity from the contralateral hemisphere for $180 \mathrm{msec}$. following the stimulus. In each patient, both methods of investigation were used, but all the records reproduced in this paper are of the second type. The averaged response was displayed on a pair of cathode ray tubes and photographed.

The system was calibrated at the beginning and end of each session by feeding in a square wave of known voltage for a known number of sweeps. In recording, the effective gain can be changed by altering the number of responses (sweeps) added together and/or by changing the charging resistor (Dawson, 1954). In the following investigation the number of responses averaged varied between 132 and 330 , depending on the degree of relaxation achieved by the patient (and hence the background noise level). Good relaxation is important for satisfactory recording of the evoked response, and in every case the patient was instructed to relax the jaw muscles and to refrain from eye movements during the actual period of the recording. In no case were we unable to obtain a number of satisfactory records. The recording session usually lasted from between three-quarters to one and a half hours.

\section{RESULTS}

CASES WITH IMPAIRED PAIN AND TEMPERATURE SENSE ALONE In the first group are patients with normal joint position sense but impaired appreciation of pin prick and temperature. Three of these patients had bilateral loss of sensation and in the other two the loss was unilateral.

Case 1 O.L., aged 49 (N.H. No. A1244), had a prolapse of the intervertebral disc between D10 and D11 with cord compression. Joint position sense was normal in both lower limbs but appreciation of pin prick and temperature was completely lost. The anterior tibial nerve was stimulated on either side and from the contralateral recording electrodes a cortical evoked potential of normal latency and amplitude was obtained (Fig. 1a).

Case 2 N.L.E., aged 11 (N.H. No. A4306), was a negro girl from the Gambia suffering from a severe diffuse spinal cord lesion of subacute onset and uncertain aetiology. The myelogram was normal. On clinical testing awareness of pin prick, heat, and cold was lost in both lower limbs, joint position sense being normal. An evoked response of normal latency and wave form was obtained from both hemispheres on stimulation of the contralateral anterior tibial nerve. However, the amplitude of the wave over the left hemisphere, although within normal limits, was smaller than that on the opposite side (Fig. 1b). On clinical testing at this time there was no detectable difference between sensation on the two sides, but the subjective threshold to electrical stimulation was definitely higher in the right leg. Three days after this recording, presumably by an extension of the lesion, she developed postural loss in the right toes and ankle joint. This persisted for about a week. It is possible that at the time of the recording there was already some minimal affection of the posterior columns on the right which did not manifest itself clinically.

Case 3 T.B., aged 19 (N.H. No. A2627), was a young American serviceman who had suffered a severe extension injury of the neck without radiological evidence of bony damage. After the accident he was found to have loss of appreciation to pin prick over the right forearm and hand and over the left hand, awareness of light touch and joint movements being unimpaired. Stimulation of the index and middle fingers of either hand produced apparently normal responses recorded over the contralateral hand area (Fig. 1c).

Thus in all these three patients with bilateral loss of pain and temperature sense the cerebral evoked potential appeared with normal latency and amplitude.

Case 4 W.D.F, aged 41 (N.H. No. 95796), had clinical and myelographic evidence of severe cervical spondylosis. He had no appreciation of pin prick, heat, or cold over the left leg but all forms of sensation over the right side of the body were unimpaired. Anterior tibial nerve stimulation on either side evoked a normal cortical response from the contralateral sensory area (Fig. 1d), though in the response following the left stimulus the second negative wave was rather larger and more prolonged.

Case 5 M.M., aged 33 (N.H. No. 100200), was a patient in whom analysis of the pathological processes was more difficult. She had been investigated in New Zealand in 1958 when she presented with a right hemiparesis associated with dysphasia and dysarthria. A vertebral arteriogram under a general anaesthetic had been attempted as a brain-stem angioma was felt to be the likely diagnosis. The investigation was unsuccessful and on coming round from the anaesthetic she was found to have developed weakness of the left arm and leg and loss of pain and temperature sense over the right leg and trunk to the level of D4, which persisted. At that time she had an exploratory cervical laminectomy which showed a bruised spinal cord but no other abnormality. On stimulation of either anterior tibial nerve an evoked response with normal latency was obtained from the contralateral hemisphere (Fig. 1e). It should be noted that the amplitude of the potential over the left hemisphere is abnormally large, of the order of 12 microvolts. On the other side the potential ( 7 microvolts) is at the upper limit of the normal range. Ignoring the significance of this enhancement for the moment, it is clear that this patient showed no diminution of the potential obtained on stimulation of the analgesic side. 

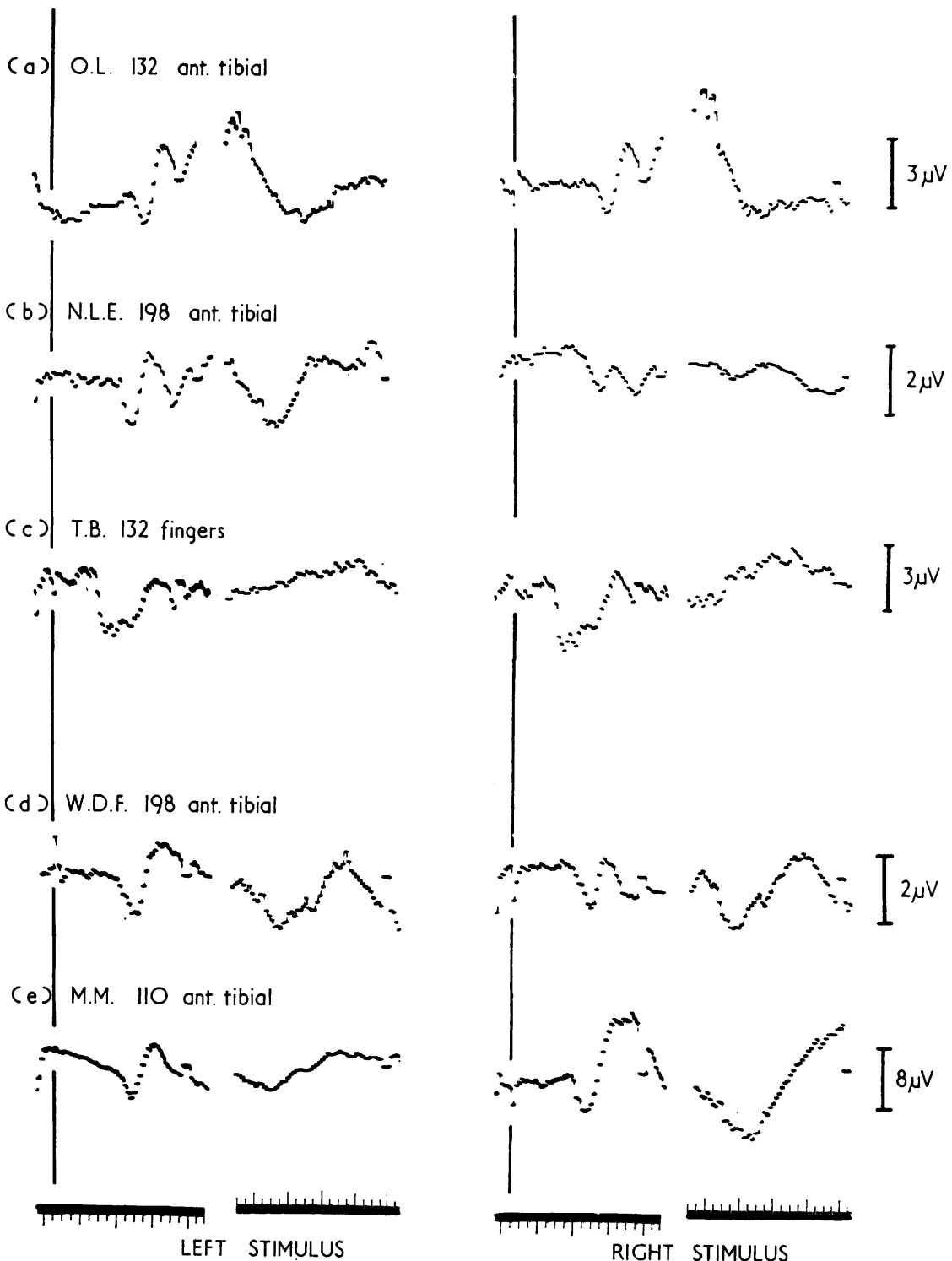

LEFT STIMULUS

RIGHT STIMULUS

FIG. 1. Records of cerebral evoked responses in three patients with bilateral (a to c) and two patients with unilateral (d to e) impairment of pain and temperature sense. The first two records in each row show the response recorded over the right postcentral gyrus to stimulation of the peripheral nerve indicated, on the left side of the body. The second two records in each row show the response recorded over the left post-central gyrus to stimulation of the nerve on the right side. Each pair of records represents the averaged response to the number of stimuli shown on the left, recorded with the two channels of the averager gated sequentially. The stimulus occurs 10 msec. after the start of the left hand record of each pair. The calibration applies to all four records in each row. Note the much smaller gains used in (e). Within the last 20 msec. of each record there are three earthed contacts which provide a voltage reference. Time scale (re-touched): 1, 5, and 20 msec. 
Considering the group as a whole, it was found that loss of pain and temperature sense in these five patients was not associated with any delay or diminution in the size of the potential recorded when the anaesthetic area was stimulated.

CASES WITH THE BROWN-SÉQUARD SYNDROME OR UNILATERAL LOSS OF JOINT POSITION SENSE The second group consists of two patients with the BrownSéquard syndrome and one with unilateral loss of joint position sense.

Case 6 G.R., aged 35 (N.H. No. A1444), had a high cervical lesion affecting the left side of the cord. After investigation the aetiology remains obscure. When we saw her she had a left hemiparesis associated with loss of joint position sense on this side together with marked impairment of pain and temperature sense on the right. On stimulation of the fingers of this right side a normal evoked response was obtained over the left hemisphere. Stimulation of the fingers on the left, however, produced no identifiable cortical response (Fig. 2a).

Case 7 V.T.E., aged 50 (N.H. No. 81531), was a West Indian negro suffering from arachnoiditis. The diagnosis was suspected on myelography and subsequently confirmed at cervical laminectomy (Mr. Harvey Jackson). Clinically he showed a classical BrownSéquard syndrome with, in the right leg, complete loss of pain and temperature sense with preservation of joint position sense and light touch and reversed findings in the opposite leg. In view of the nature of the pathological process the possibility that the sensory roots were also affected must be borne in mind. The subjective electrical threshold was extremely high and was only reached using a stimulus of longer duration than normal $(1.25 \mathrm{msec}$. time constant). Using the maximum stimulus available to us we were unable to record an evoked response over the right hemisphere following stimulation of the left anterior tibial nerve. On the other side a small cortical response was recorded (Fig. 2b). Thus although the pathological changes are less clear-cut in this case the absence of the evoked response again parallels the loss of posterior column type function.

Case 8 A.B., aged 19 (N.H. No. 93102), had been admitted with a severe brain-stem disturbance of acute onset associated with subarachnoid haemorrhage. No vertebral arteriogram was done as it was felt that the diagnosis of a brain-stem vascular anomaly could be made without reasonable doubt. At the time of his acute illness appreciation of all sensory modalities had been severely impaired over the right side of the body. At the time of recording, while joint position sense was still lost in the right arm and hand, appreciation of pain and temperature had returned to normal. Stimulation of the fingers of the right hand produced a small, very delayed and ill-formed response, the latency being approximately $45 \mathrm{msec}$. The potential recorded over the right hemisphere following stimulation of the left fingers had a normal latency and was unusually large ( 7 microvolts peak-to-peak) (Fig. 2c).

The three patients in this group showed absence or gross disturbance of the evoked response only on the side corresponding to the loss of joint position sense.

CASES WITH BILATERALLY IMPAIRED JOINT POSITION SENSE ALONE The three patients in the next group

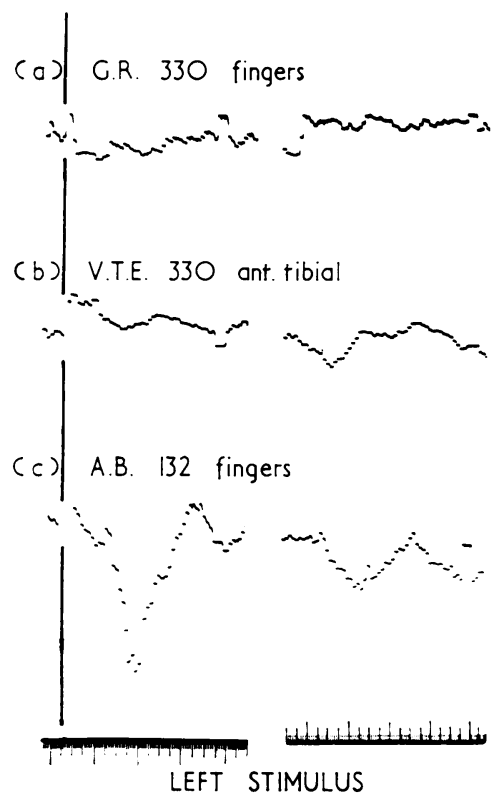

LEFT STIMULUS

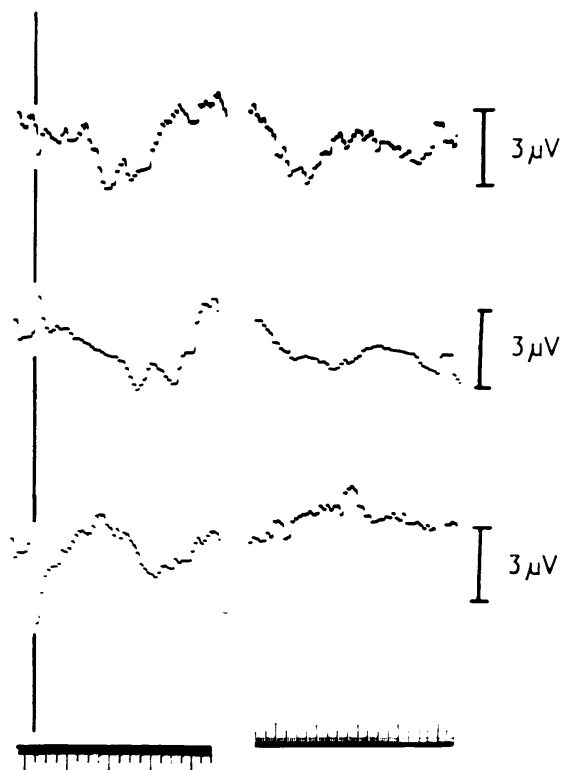

FIG. 2. Records showing the absence or diminution of the contralateral cerebral responses to peripheral nerve stimulation on the affected side in three cases with unilateral impairment of joint position sense (for further details see text). Method of recording, time scale, and calibration as described for Fig. 1. 
had varying degrees of loss of joint position sense with preservation of pin prick and temperature appreciation.

Case 9 C.K., aged 18 (N.H. No. 60284), was suffering from disseminated sclerosis and had mildly impaired postural sense in both hands, the right being more affected than the left. Stimulation of the fingers did not evoke any recognizable early potential on either side, but there was a late response which was larger over the right hemisphere on stimulation of the less affected left side (Fig. 3a). This finding, in association with mild bilateral impairment of posterior column function, suggests that the late components of the response may be more resistant to moderate disturbance of the posterior column pathways.

Case 10 A.V.G., aged 59 (N.H. No. A1019), in whom the diagnosis of disseminated sclerosis had been made, had gross impairment of postural sense in the left hand but only a slight similar defect in the right. Although stimulation of the fingers on either side produced contralateral cortical responses these were abnormal in form and the long latencies were more characteristic of those to be expected with foot stimulation. If anything the amplitude of the response was smaller on stimulation of the more severely affected left side (Fig. 3b).

Case 11 L.S.D., aged 56 (N.H. No. A3680), had severe loss of posterior column function due to a thoracic meningioma. The recordings were made pre-operatively. Joint position sense was grossly impaired in both feet, appreciation of other modalities being normal. Any response following very strong stimulation of the right anterior tibial nerve was barely detectable. Stimulation of the left side evoked a surface positive wave over the right hemisphere with an amplitude of about 2 microvolts and a peak positivity at $95 \mathrm{msec}$. and there was complete absence of the early components of the response (Fig. 3c).

All three patients in this group showed small abnormal responses with an absence of early components and long latencies to the first identifiable response.

CASES SHOWING PARTIAL RECOVERY AFTER LOSS OF JOINT-POSITION SENSE The last group consists of three patients who had suffered from fairly severe loss of postural sense from which they had recovered either completely or markedly at the time of recording.

Case 12 M.H., aged 49 (N.H. No. 98237), was recorded six months after a thoracic meningioma had been removed by Mr. Harvey Jackson. At operation the tumour was found to be tightly adherent to the posterior columns and great difficulty was encountered in removing it. In the immediate post-operative period there was complete loss of joint position sense in both limbs but pin prick, heat, and cold were appreciated normally. When we saw her recovery had occurred to the extent that she could tell accurately when her toes were moved but she was wrong about the direction of movement five times out of ten. Both sides appeared equally affected. It should be noted that the subjective electrical threshold was very much raised (Table II). Anterior tibial nerve stimulation failed to evoke any clear response using the maximum output of the stimulator (Fig. 4a).
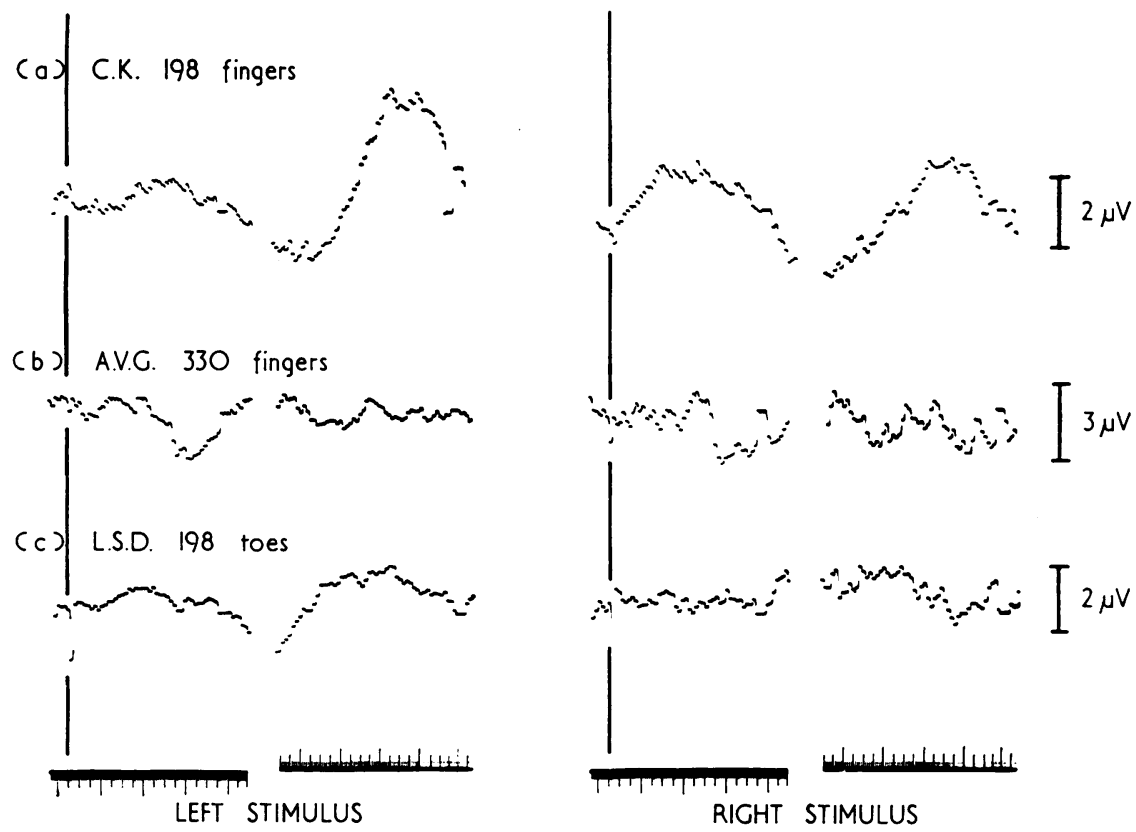

FIG. 3. Records showing the abnormal, delayed, or absent contralateral cerebral responses to peripheral nerve stimulation in three cases with bilateral impairment of joint position sense. For further details see Fig. 1 and text. 
Case 13 F.P., aged 64 (N.H. No. A2010), before removal of a thoracic meningioma (Mr. Harvey Jackson), had had impairment of joint position sense in both lower limbs, the left side being the more affected. We saw her three weeks post-operatively when it was not possible to fault her postural sense in the toes of either foot. We only recorded from the right hemisphere in this patient, stimulating the left anterior tibial nerve. A strong stimulus produced only a very small response, the sum of 198 consecutive stimuli. This patient was the only one in this group to show a well-formed late wave (Fig. 4b).

Case 14 J.M., aged 55 (N.H. No. A3847), was suffering from cervical spondylosis and had such slight disturbance of postural sense that he missed only an occasional small movement of the right hallux. Pain and temperature sense were impaired, but not lost, in both legs. He said that five weeks previously his legs had been more numb. This had been three weeks before admission and no clinical testing had been carried out at this time, so that the evidence of recovery of posterior column-type sensation in this case is only circumstantial. Right anterior tibial nerve stimulation produced no cortical evoked potential while stimulation of the left side, where postural sense was clinically normal, produced a small abnormal and delayed response (Fig. 4c) resembling closely that shown by the last patient (case 11) of the previous group.

Thus the three patients in this group showed no cortical responses where there was mild residual impairment of posterior column function and extremely small, abnormal responses where full clinical recovery had occurred.

\section{DISCUSSION}

The findings in the whole series are summarized in Table II. In the first group loss of pain and temperature sense was associated with a normal evoked response on stimulation of the affected area. There are only two anomalies. The smaller response from the left hemisphere of case 2 , in which there was definite clinical evidence of some posterior column involvement on the right side soon after our recording, and the much enhanced responses in case 5 .

In the second group, unilateral loss of joint position sense was associated with the response being either lost or delayed and diminished on the corresponding side. In case 7 , a patient who had arachnoiditis and very high electrical thresholds, the clinically definite Brown-Séquard syndrome did not imply that fine discriminative sensation was completely unaffected on the right side, and this is borne out by the small potential recorded following the right stimulus. However, the difference between the potential on the two sides is clear cut and follows the clinical picture. In case 8, the patient again acts as his own control. There is a very large response, possibly enhanced, on the clinically unaffected side, whereas the response on the other side is delayed, ill-formed, and less than a third of the size.

In the third group the cerebral response is in all cases lost or abnormal and the degree of abnormality
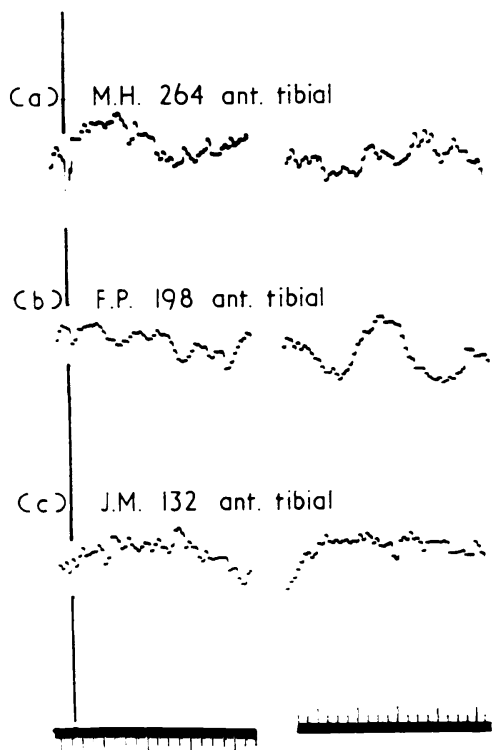

LEFT STIMULUS

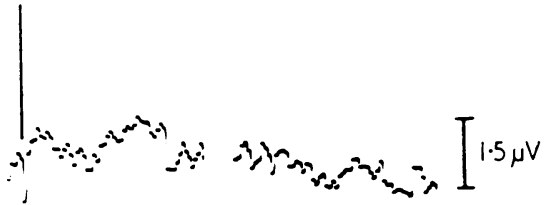

FIG. 4. Records showing diminished or absent contralateral cerebral responses to $\left[2 \mu \mathrm{V} \begin{array}{l}\text { peripheral nerve } \\ \text { stimulation in three }\end{array}\right.$ cases with complete or partial recovery of sensation following severe bilateral impairment of joint position sense. For further details see Fig. 1 and text. 
TABLE II

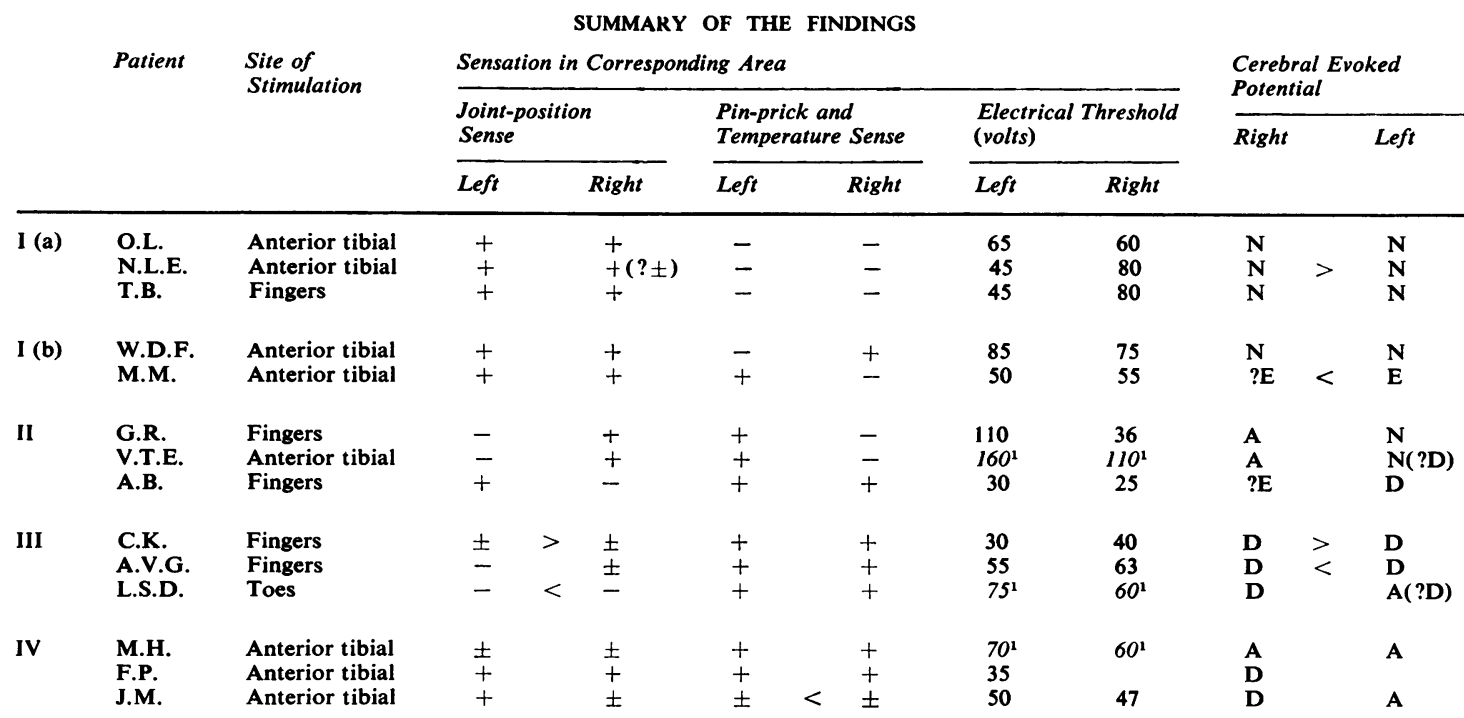

\footnotetext{
+ Normally appreciated \pm Slightly impaired - Grossly impaired ${ }^{1} 0.25 \mu \mathrm{F}$ capacitor. All other readings were with $0.1 \mu \mathrm{F}$ capacitor

N Apparently normal potential with usual latency
}

D Diminished or delayed potential

A Absent potential

E Unusually large potential ? enhanced appears to be related to the severity of the loss of the sensation subserved by the posterior column pathways. The large late wave recorded in case 9 , but not in the other two cases, may have been related to the mildness of the sensory loss shown by this patient. It may be that moderate impairment is associated with delayed conduction and an increased scatter of a normally synchronous ascending volley, so that the form of the early potential is broken up and the response occurs with a longer latency.

The results obtained with the patients in the fourth group show, however, that the correlation of evoked potential changes with the severity of clinical sensory loss does not apply to patients showing complete or nearly complete clinical recovery of joint position sense. The evoked response may remain absent or grossly diminished at a time when clinical testing reveals no residual deficit. This is analogous to the findings with regard to the nerve action potential following recovery from peripheral neuritis. The potential may be absent or grossly abnormal at a time when clinical recovery has taken place.

All these results are consistent with the suggestion of Giblin (1960) that the cerebral evoked response depends on the integrity of the posterior-column pathways, but is unaffected by lesions of the spinothalamic tracts. There is no evidence that there is any component of the response, which is attenuated in the patients showing loss of pain or temperature sense Conversely, even the late components appear to be lost in cases with severe loss of sensation of posterior column type (cases 11, 12, and 14) which suggests that there is no late component dependent on the more slowly conducting anterior column pathways. We have thus no evidence to support the suggestion of Alajouanine et al. that some of the afferent impulses responsible for the cortical potential travel by the spinothalamic tracts.

Two of the patients in this series had unusually large responses on one side. The potential recorded from the left hemisphere in case 5 on stimulation of the analgesic foot is well outside the range of amplitudes seen in normal subjects. The right hemisphere potential is also very large, though it is probably just within the limits of normal. In case 8 the amplitude of the response was large on stimulation of the clinically normal hand, the other hand showing loss of joint position sense. In both these patients there was undoubted evidence of a brainstem lesion.

Abnormally large cerebral evoked responses are seen in myoclonic epilepsy or in the 'myoclonus' secondary to cerebral lipoidosis with widespread cortical destruction. We have also noted this enhancement phenomenon in a number of other patients with brain-stem lesions, with or without 
associated sensory loss, and these findings will be published in detail elsewhere (Halliday and Wakefield, 1963).

\section{SUMMARY}

A consecutive series of 14 patients with dissociated sensory loss has been examined clinically, and their cerebral evoked responses to electrical stimulation of either the hand or foot have been recorded for the $180 \mathrm{msec}$. immediately following the stimulus. The evoked response was found to be unaffected by lesions causing loss of pin-prick or temperature sense, but was lost, or diminished and delayed, where there was loss of joint position sense. It is concluded that the impulses responsible for both the early and late components of the evoked response travel by the posterior column pathways. Two patients of the series, who had brain-stem lesions, had abnormally large responses.

We should like to thank Dr. W. A. Cobb for providing us with facilities in his department, and the physicians of the National Hospital who allowed us to examine their patients. Our thanks are especially due to Mr. H. B. Morton for his technical assistance.

\section{REFERENCES}

Alajouanine, Th., Scherrer, J., Barbizet, J., Calvet, J., and Verley, R. (1958). Rev. neurol., 98, 757.

Dawson, G. D. (1947a). J. Neurol. Neurosurg. Psychiat., 10, 137. (1947b). Ibid., 10, 141.

- (1954). Electroenceph. clin. Neurophysiol., 6, 65.

Giblin, D. R. (1960). lbid., 12, 262.

Halliday, A. M., and Wakefield, G. S. (1962). Ibid., 14, 786. (1963). To be published.

Rose, J. E., and Mountcastle, V. B. (1959). In Handbook of Physiology, edited by H. W. Magoun. Section 1. Neurophysiology, vol. I, p.387. American Physiological Society, Washington, D.C. 